\title{
Concise Asymmetric Formal Synthesis of Pyrrolopiperazinone Natural Products by Tandem Cross Metathesis/Intramolecular Aza-Conjugate Addition
}

\author{
Su-Hyun Kwon, Hyo-Jun Lee, and Chang-Woo Cho* \\ Department of Chemistry, Kyungpook National University, Daegu 702-701, Korea. *E-mail: cwcho@knu.ac.kr \\ Received October 18, 2010, Accepted October 26, 2010
} Key Words: Pyrrolopiperazinone, Formal synthesis, Tandem reaction, Olefin cross metathesis, Intramolecular
aza-conjugate addition

With the advent of air-stable, commercially available, and functional group-tolerable ruthenium-carbene catalysts, olefin metathesis has become a powerful tool for carbon-carbon double bond formations, with various applications reported over the past decade. ${ }^{1}$ Meanwhile, tandem reactions, which involve ruthenium-catalyzed olefin metathesis followed by other transformations, have also been reported, giving non-metathetic products. ${ }^{2}$ These tandem reactions broaden the synthetic utility of metathesis-active ruthenium-carbene catalysts beyond olefin metathesis, and provide an important tool for the rapid and efficient synthesis of complex molecules from simple starting materials. Recent examples include ring-closing metathesis (RCM) with Kharasch addition, ${ }^{3}$ olefin isomerization, ${ }^{4}$ oxidation process, ${ }^{5}$ and hydrogenation ${ }^{6}$ cross metathesis (CM) with intramolecular aza-Michael reaction, ${ }^{7}$ intramolecular oxa-conjugate cyclization, ${ }^{8}$ and intramolecular hydroarylation; ${ }^{9}$ and ring-closing enyne metathesis (RCEYM) with cyclopropanation, ${ }^{10}$ metallotropic [1,3]-shift, ${ }^{11}$ and hydrovinylation. ${ }^{12}$ In these tandem reactions, the use of $N$-centered heteroaromatic nucleophiles remains unexplored, in contrast to the reported use of an $\mathrm{N}$-centered aliphatic nucleophile in the tandem $\mathrm{CM} /$ intramolecular aza-Michael reaction, ${ }^{7}$ in spite of the importance of optically pure $N$-heteroaromatic compounds as pharmacophores in biologically active natural products. ${ }^{13}$ Therefore, we planned an asymmetric formal synthesis of pyrrolopiperazinone natural product via the development of stereoselective tandem $\mathrm{CM}$ /
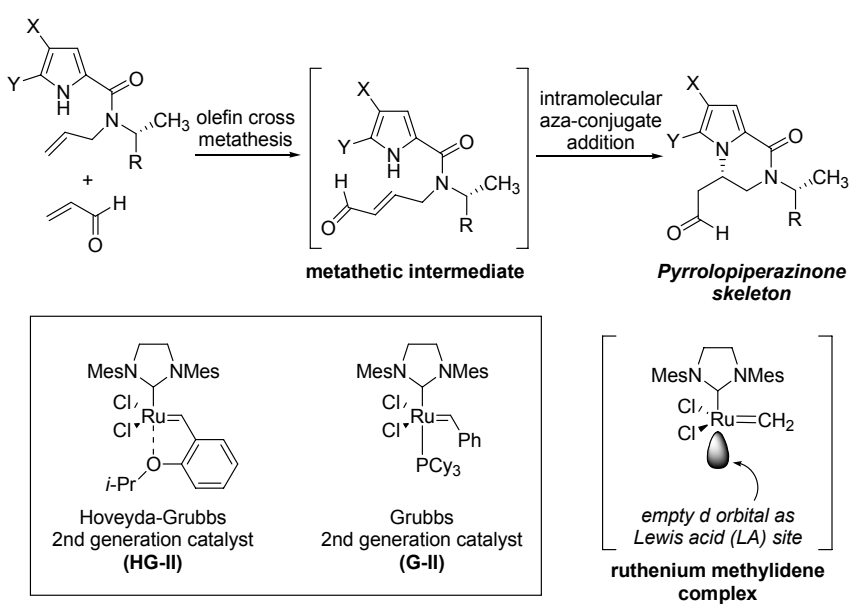
Pyrrolopiperazinone skeleton

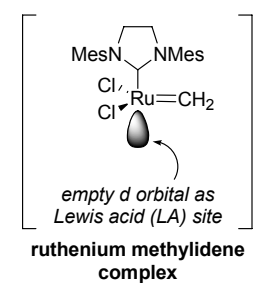

Scheme 1. Concept of metathesis-active ruthenium-catalyzed diastereoselective tandem reaction for the formation of pyrrolopiperazinone skeleton intramolecular aza-conjugate addition reactions using pyrrole as the first use of the $N$-centered heteroaromatic nucleophile in the metathesis-active ruthenium-catalyzed tandem reactions (Scheme 1). Moreover, it was supposed that the tandem reaction would be performed by the sequential activation of the metathetic intermediate with an active ruthenium species as a Lewis acid, e.g., a ruthenium methylidene complex ${ }^{7,10}$ generated from the initiation of the CM process, or a ruthenium hydride species ${ }^{8,14}$ generated from the decomposition of the ruthenium methylidene complex under high temperature conditions.

Pyrrolopiperazinone is one of the key skeletons found in pyrrole alkaloids, which are a class of marine natural products with a variety of potent biological activities. ${ }^{15}$ Examples of pyrrolopiperazinone natural products include hanishin, ${ }^{16}$ longamide $\mathrm{B},{ }^{17}$ longamide B methyl ester, ${ }^{18}$ cyclooroidin, ${ }^{19}$ and the agesamides $^{20}$ (Figure 1). They were isolated from sponge Agelas species, except for hanishin, which came from Acanthella species, and exhibit interesting biological properties such as cytotoxic activity and antibiotic activity. Although these pyrrolopiperazinone natural products have considerable biological activities and chemical skeletons, and thus are potentially useful for the development of pharmaceuticals, few reports on the asymmetric syntheses of the above pyrrolopiperazinone natural products are available. The only reports involve synthetic routes using Pd-catalyzed asymmetric annulation ${ }^{21}$ and chiral pool ${ }^{22}$ for the asymmetric synthesis of the pyrrolopiperazinone natural products. ${ }^{23}$ Therefore, the development of a new route for the asymmetric synthesis of the pyrrolopiperazinone natural products is highly desirable. Herein, we report the concise asymmetric formal synthesis of the pyrrolopiperazinone natural products via the diastereoselective tandem $\mathrm{CM} /$ intramolecular aza-conjugate addition of acrolein to substrates $\mathbf{3}$ having pyrrole as the $N$-centered heteroaromatic nucleophile (Scheme 2).

To explore the feasibility of using the diastereoselective
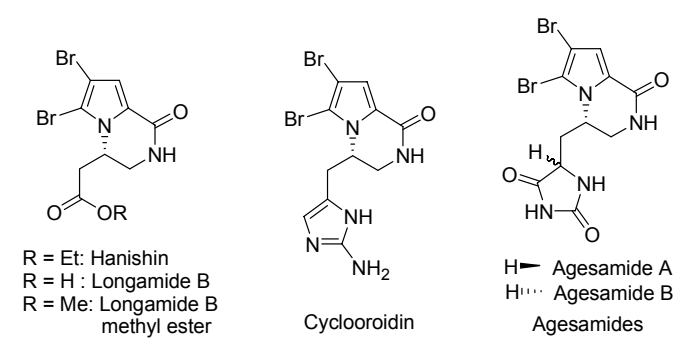

Figure 1. Pyrrolopiperazinone natural products 


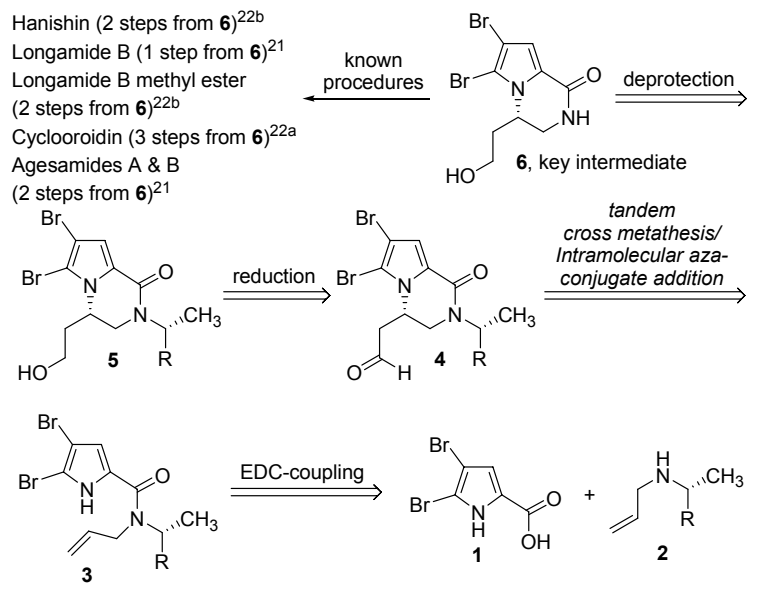

Scheme 2. Retrosynthetic analysis for asymmetric formal synthesis of pyrrolopiperazinone natural products

$$
\begin{aligned}
& \text { 3a, } \mathrm{R}=\mathrm{Ph}, 81 \% \text { Yield } \\
& \mathbf{3 b}, \mathrm{R}=1-\mathrm{Naph}, 84 \% \text { Yield }
\end{aligned}
$$

Scheme 3. Preparation of substrates $\mathbf{3 a}$ and $\mathbf{3 b}$

metathesis-active ruthenium-catalyzed tandem $\mathrm{CM}$ /intramolecular aza-conjugate addition as the key reaction for the asymmetric formal synthesis of the pyrrolopiperazinone natural products, we first prepared substrates 3 bearing $(R)$-1-arylethyl groups as a chiral auxiliary to control the asymmetric induction in the tandem reactions (Scheme 3). The substrates $\mathbf{3 a}$ and $\mathbf{3 b}$ were obtained by amide bond forming reactions of commercially available 4,5-dibromo- $1 H$-pyrrole-2-carboxylic acid (1) with a series of $(R)-N$-allyl-1-arylethylamines $\mathbf{2 a}$ and $\mathbf{2 b}$ using EDC.HCl-DMAP as coupling reagents and HOBt as a deracemization additive. Good yields were obtained without any racemization, as determined by chiral HPLC analysis (Chiralcel OD-H) with a racemic standard.

With the substrates $\mathbf{3 a}$ and $\mathbf{3 b}$ prepared, the diastereoselective tandem $\mathrm{CM} /$ intramolecular aza-conjugate addition reactions of acrolein to the substrates 3 using Hoveyda-Grubbs 2nd generation catalyst (HG-II) in dichloromethane at ambient temperature were carried out (Table 1, entries 1-2). Among the substrates $\mathbf{3 a}$ and $\mathbf{3 b}$ tested, gratifyingly, substrate $\mathbf{3 b}$, bearing the bulkier chiral $(R)$-1-(1-naphthyl)ethyl group as the chiral auxiliary, provided the corresponding tandem product $4 \mathrm{~b}$ in $55 \%$ yield as a 2.3:1 mixture of diastereomers, without the metathetic intermediate. When HG-II was replaced with Grubbs 2nd generation catalyst $(\mathbf{G}-\mathbf{I I})$ at $40{ }^{\circ} \mathrm{C}$, with the other reaction conditions remaining the same, the tandem product $\mathbf{4 b}$ was obtained with a decreased yield of $32 \%$ and diastereomeric ratio of $1.3: 1$ (Table 1, entry 3 vs. entry 2). Hence, HG-II proved superior. By varying the reaction temperature to increase the yield and the diastereomeric ratio of the tandem reaction, ambient temperature was identified as the ideal temperature for the tandem reaction (Table 1 , entry $4 v s$. entry 2 ). Then, using a portionwise addition method for HG-II (Table 1, entries 5-6), indeed, when
Table 1. Optimization of diastereoselective tandem CM/intramolecular aza-conjugate addition reactions of acrolein to substrates $\mathbf{3}^{a}$

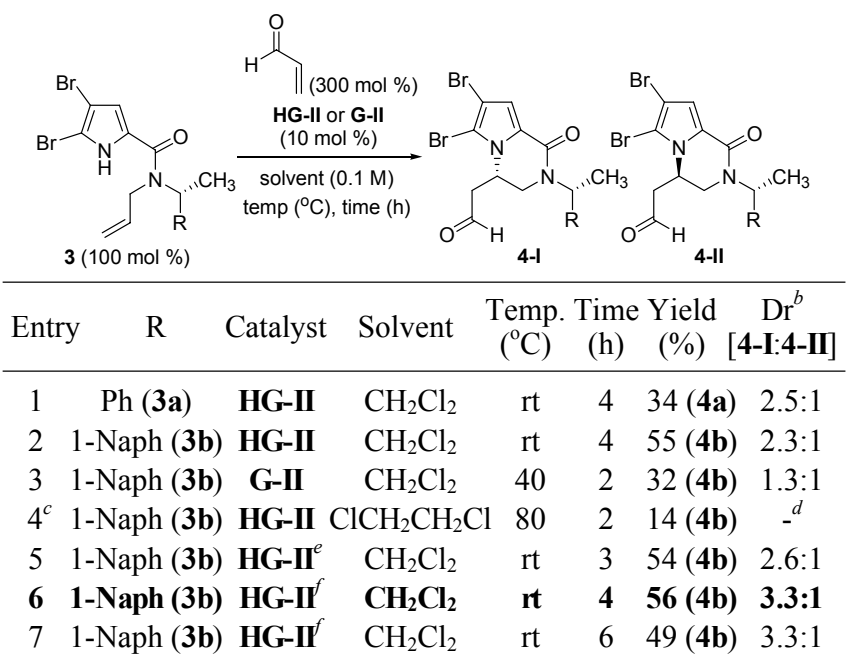

${ }^{a}$ Procedure: To a solution of substrate $\mathbf{3}(0.3 \mathrm{mmol}, 100 \mathrm{~mol} \%)$ and catalyst $(0.03 \mathrm{mmol}, 10 \mathrm{~mol} \%)$ in $\mathrm{CH}_{2} \mathrm{Cl}_{2}(3 \mathrm{~mL}, 0.1 \mathrm{M})$ was added acrolein $(0.9$ $\mathrm{mmol}, 300 \mathrm{~mol} \%$ ). The reaction mixture was stirred at $\mathrm{rt}, 40^{\circ} \mathrm{C}$, or $80{ }^{\circ} \mathrm{C}$, until the substrate was no longer consumed, at which point the reaction mixture was evaporated onto silica gel and the product was isolated by silica gel chromatography. ${ }^{b}$ Dr was determined by isolated yields of two diastereomers. ${ }^{c}$ Crotonaldehyde instead of acrolein was used. ${ }^{d}$ Not determined. ${ }^{e}$ Two $5 \mathrm{~mol} \%$ additions of HG-II were used, with an interval of one hour. ${ }^{f}$ Two $5 \mathrm{~mol} \%$ additions of HG-II were used, with an interval of two hours.

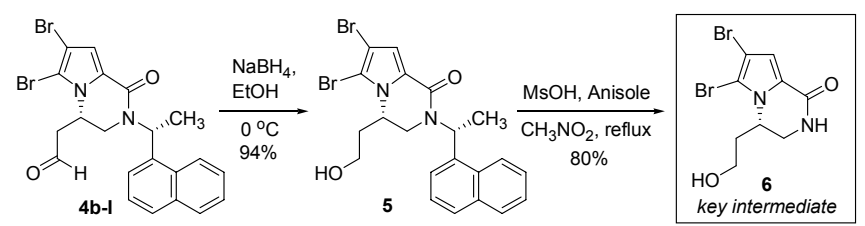

Scheme 4. Formal synthesis of key intermediate 6 in synthesis of pyrrolopiperazinone natural products

the two $5 \mathrm{~mol} \%$ additions of HG-II were carried out at an interval of two hours in the tandem reaction, the tandem product $4 \mathrm{~b}$ was obtained in $56 \%$ yield and an increased diastereomeric ratio of 3.3:1 (Table 1, entry 6). In cases where the reaction with the intervals of two hours for the HG-II additions was carried out longer than four hours, no further increase in the yield of $\mathbf{4 b}$ was obtained (Table 1 , entry 7 ).

To complete the asymmetric formal synthesis of the pyrrolopiperazinone natural products, reduction of the tandem product 4b-I was performed with sodium borohydride in ethanol at $0{ }^{\circ} \mathrm{C}$, affording the alcohol 5 in 94\% yield (Scheme 4). Finally, the removal of the $(R)$-1-(1-naphthyl)ethyl group of 5 with methanesulfonic acid and anisole in nitromethane at reflux provided the useful intermediate 6 in the synthesis of the pyrrolopiperazinone natural products, as reported earlier, in $80 \%$ yield. The spectroscopic and analytical data for $\mathbf{6}$ were in full agreement with those reported. ${ }^{21,22 b}$ The pyrrolopiperazine natural products such as hanishin, ${ }^{22 \mathrm{~b}}$ longamide $\mathrm{B},{ }^{21}$ longamide B methyl ester, ${ }^{22 \mathrm{~b}}$ cyclooroidin, ${ }^{22 a}$ and the agesamides ${ }^{21}$ can be synthesized from the intermediate $\mathbf{6}$ in a few steps according to procedures found in the literature.

In summary, we achieved the concise asymmetric formal synthesis of the pyrrolopiperazinone natural products, via the 
diastereoselective tandem $\mathrm{CM}$ /intramolecular aza-conjugate addition reaction, from 4,5-dibromo- $1 \mathrm{H}$-pyrrole-2-carboxylic acid (1) to the key intermediate $\mathbf{6}$ in only four linear steps. The tandem reaction of substrate $\mathbf{3} \mathbf{b}$, bearing the $(R)-1$-(1-naphthyl)ethyl group as the chiral auxiliary, was performed using HG-II as a single species to carry out the sequential reaction, affording a $56 \%$ yield of the non-metathetic product, pyrrolopiperazinone $4 \mathbf{b}$, as a 3.3:1 mixture of diastereomers. In addition, this is the first example of pyrroles as an $\mathrm{N}$-centered heteroaromatic nucleophile in the metathesis-active ruthenium-catalyzed tandem reactions. Future studies will be devoted to the development of new metathesis-active ruthenium-catalyzed tandem reactions and their application to the synthesis of biologically active natural products.

\section{Experimental Section}

General Procedure for the Synthesis of Substrates 3a and 3b. To a stirred solution of 4,5-dibromo- $1 H$-pyrrole-2-carboxylic acid (1, $2.84 \mathrm{~g}, 10.59 \mathrm{mmol}),(R)-N$-allyl-1-arylethylamine 2 (3.53 mmol), 1-hydroxybenzotriazole $(1.43 \mathrm{~g}, 10.59 \mathrm{mmol})$, and 4-DMAP $(1.29 \mathrm{~g}, 10.59 \mathrm{mmol})$ in DMF $(12 \mathrm{~mL})$ was added $N$-(3-dimethylaminopropyl)- $N$ '-ethylcarbodiimide hydrochloride $(2.03 \mathrm{~g}, 10.59 \mathrm{mmol})$ at ambient temperature. The mixture was heated to $80^{\circ} \mathrm{C}$ and stirred for $12 \mathrm{~h}$. The mixture was quenched with $1.0 \mathrm{M} \mathrm{HCl}$ solution and extracted with $\mathrm{Et}_{2} \mathrm{O}$. The combined ether layers were washed with saturated $\mathrm{NaHCO}_{3}$ followed by brine, and dried over $\mathrm{Na}_{2} \mathrm{SO}_{4}$. The solvent was evaporated in vacuo to give the crude product.

(R)- $\mathrm{N}$-Allyl-4,5-dibromo- $\mathrm{N}$-(1-phenylethyl)- $1 \mathrm{H}$-pyrrole2-carboxamide (3a). Prepared by the general procedure from (R)-N-(1-phenylethyl)prop-2-en-1-amine (2a, 0.569 g, 3.53 $\mathrm{mmol})$. The crude product was purified by flash chromatography $\left(\mathrm{SiO}_{2}, 5 \%\right.$ EtOAc/hexanes) to give the substrate 3a as a light brown oil (1.17 g, 81\%): $[\alpha]_{\mathrm{D}}^{20}+63.5\left(c 1, \mathrm{CH}_{3} \mathrm{OH}\right) ;{ }^{1} \mathrm{H}$ NMR $\left(400 \mathrm{MHz}, \mathrm{CDCl}_{3}\right) \delta 11.57(\mathrm{~s}, 1 \mathrm{H}), 7.35-7.25(\mathrm{~m}, 5 \mathrm{H}), 6.60(\mathrm{~d}$, $J=3.2 \mathrm{~Hz}, 1 \mathrm{H}), 6.20-6.15(\mathrm{~m}, 1 \mathrm{H}), 5.87-5.75(\mathrm{~m}, 1 \mathrm{H}), 5.10-5.06$ $(\mathrm{m}, 2 \mathrm{H}), 4.21(\mathrm{~d}, J=16.8 \mathrm{~Hz}, 1 \mathrm{H}), 3.82-3.67(\mathrm{~m}, 1 \mathrm{H}), 1.65(\mathrm{~d}$, $J=6.8 \mathrm{~Hz}, 3 \mathrm{H}) ;{ }^{13} \mathrm{C} \mathrm{NMR}\left(100 \mathrm{MHz}, \mathrm{CDCl}_{3}\right) \delta 161.1,140.0$, $134.7,128.5,127.5,127.3,125.8,116.7,115.5,105.9,99.5$, 53.5, 46.5, 17.5; IR (neat) 3168, 2925, 1588, 1451, 1412, 1240, $1177,976,751,698 \mathrm{~cm}^{-1}$; HRMS calcd for $[\mathrm{M}] \mathrm{C}_{16} \mathrm{H}_{16} \mathrm{Br}_{2} \mathrm{~N}_{2} \mathrm{O}$ 409.9629, found 409.9626.

(R)- $N$-Allyl-4,5-dibromo- $N$-(1-(naphthalen-1-yl)ethyl)$1 H$-pymole-2-carboxamide (3b). Prepared by the general procedure from $(R)-N$-(1-(naphthalene-1-yl)ethyl)prop-2-en-1amine (2b, $0.745 \mathrm{~g}, 3.53 \mathrm{mmol})$. The crude product was purified by flash chromatography $\left(\mathrm{SiO}_{2}, 5 \% \mathrm{EtOAc} /\right.$ hexanes $)$ to give the substrate $\mathbf{3 b}$ as a light yellow solid $(1.37 \mathrm{~g}, 84 \%)$ : $\mathrm{mp} 79-81^{\circ} \mathrm{C}$; $[\alpha]_{\mathrm{D}}^{20}+31.4\left(c 1, \mathrm{CH}_{3} \mathrm{OH}\right) ;{ }^{1} \mathrm{H}$ NMR $\left(400 \mathrm{MHz}, \mathrm{CDCl}_{3}\right) \delta 10.66$ $(\mathrm{s}, 1 \mathrm{H}), 8.05-7.95(\mathrm{~m}, 1 \mathrm{H}), 7.86-7.82(\mathrm{~m}, 2 \mathrm{H}), 7.59-7.57(\mathrm{~m}$, $1 \mathrm{H}), 7.49-7.46(\mathrm{~m}, 3 \mathrm{H}), 6.82(\mathrm{~s}, 1 \mathrm{H}), 6.55-6.45(\mathrm{~m}, 1 \mathrm{H}), 5.32-$ $5.21(\mathrm{~m}, 1 \mathrm{H}), 4.70-4.64(\mathrm{~m}, 2 \mathrm{H}), 3.98-3.81(\mathrm{~m}, 2 \mathrm{H}), 1.75(\mathrm{~d}$, $J=5.2 \mathrm{~Hz}, 3 \mathrm{H}) ;{ }^{13} \mathrm{C} \mathrm{NMR}\left(100 \mathrm{MHz}, \mathrm{CDCl}_{3}\right) \delta 160.4,135.1$, 134.2, 133.6, 132.3, 129.0, 128.6, 126.9, 125.9, 125.1, 124.8, 123.6, 116.2, 115.7, 106.1, 99.6, 49.8, 46.2, 16.9; IR (neat) 3169, 2929, 1580, 1458, 1413, 1236, 1177, 975, $780 \mathrm{~cm}^{-1}$; HRMS calcd for $[\mathrm{M}] \mathrm{C}_{20} \mathrm{H}_{18} \mathrm{Br}_{2} \mathrm{~N}_{2} \mathrm{O} 459.9786$, found 459.9785 .
2-((S)-6,7-Dibromo-1-oxo-2-((R)-1-phenylethyl)-1,2,3,4tetrahydropyrrolo $[1,2-a]$ pyrazin-4-yl)acetaldehyde (4a). Dichloromethane $(2 \mathrm{~mL}, 0.1 \mathrm{M})$, acrolein $(40 \mu \mathrm{L}, 0.6 \mathrm{mmol})$ and Hoveyda-Grubbs 2nd generation catalyst $(12.5 \mathrm{mg}, 0.02 \mathrm{mmol}$ ) were added to a $10 \mathrm{~mL}$ round-bottomed flask charged with $(R)$ - $N$-allyl-4,5-dibromo- $N$-(1-phenylethyl)-1 $H$-pyrrole-2-carboxamide (3a, $82 \mathrm{mg}, 0.2 \mathrm{mmol}$ ) at room temperature and the mixture was stirred for 4 hours. After that time, the solution was concentrated to dryness and purified by flash chromatography $\left(\mathrm{SiO}_{2}, 20 \% \mathrm{EtOAc/hexanes)}\right.$ to provide $\mathbf{4 a - I}$ as major diastereomer in $24 \%$ yield ( $21 \mathrm{mg}$, a light brown solid), and 4a-II as minor diastereomer in 10\% yield ( $9 \mathrm{mg}$, a yellow solid), respectively (total yield: 34\%):

4a-I as Major Diastereomer: $\operatorname{mp} 118-119^{\circ} \mathrm{C} ;[\alpha]_{\mathrm{D}}^{20}-221.9$ (c $\left.1.15, \mathrm{CH}_{3} \mathrm{OH}\right) ;{ }^{1} \mathrm{H} \mathrm{NMR}\left(400 \mathrm{MHz}, \mathrm{CDCl}_{3}\right) \delta 9.79(\mathrm{~s}, 1 \mathrm{H})$, $7.35-7.26(\mathrm{~m}, 5 \mathrm{H}), 7.02(\mathrm{~s}, 1 \mathrm{H}), 6.16(\mathrm{q}, J=7.2 \mathrm{~Hz}, 1 \mathrm{H}), 4.75-$ $4.73(\mathrm{~m}, 1 \mathrm{H}), 3.40-3.30(\mathrm{~m}, 2 \mathrm{H}), 3.04(\mathrm{dd}, J=18.8,10.4 \mathrm{~Hz}$, $1 \mathrm{H}), 2.75$ (dd, $J=17.6,1.2 \mathrm{~Hz}, 1 \mathrm{H}), 1.48(\mathrm{~d}, J=6.8 \mathrm{~Hz}, 3 \mathrm{H})$; ${ }^{13} \mathrm{C} \mathrm{NMR}\left(100 \mathrm{MHz}, \mathrm{CDCl}_{3}\right) \delta 198.1,157.1,138.8,128.6,127.8$, 127.2, 125.4, 116.3, 105.0, 101.1, 48.8, 48.3, 44.7, 43.2, 16.0; IR (neat) 2926, 1720, 1640, 1426, 1329, 1129, 967, $700 \mathrm{~cm}^{-1}$; HRMS calcd for [M] $\mathrm{C}_{17} \mathrm{H}_{16} \mathrm{Br}_{2} \mathrm{~N}_{2} \mathrm{O}$ 437.9579, found 437.9580:

4a-II as Minor Diastereomer: $\operatorname{mp} 71-73{ }^{\circ} \mathrm{C}$; $[\alpha]_{\mathrm{D}}^{20}-207.6(\mathrm{c}$ $\left.1.1, \mathrm{CH}_{3} \mathrm{OH}\right) ;{ }^{1} \mathrm{H} \mathrm{NMR}\left(400 \mathrm{MHz}, \mathrm{CDCl}_{3}\right) \delta 8.87(\mathrm{~s}, 1 \mathrm{H}), 7.38-$ $7.26(\mathrm{~m}, 5 \mathrm{H}), 7.02(\mathrm{~s}, 1 \mathrm{H}), 6.11(\mathrm{q}, J=7.2 \mathrm{~Hz}, 1 \mathrm{H}), 4.63-4.59$ (m, $1 \mathrm{H}), 3.74$ (ddd, $J=13.2,4.0,1.2 \mathrm{~Hz}, 1 \mathrm{H}), 3.29$ (dd, $J=13.2$, $1.2 \mathrm{~Hz}, 1 \mathrm{H}), 2.39-2.31(\mathrm{~m}, 2 \mathrm{H}), 1.53(\mathrm{~d}, J=7.2 \mathrm{~Hz}, 3 \mathrm{H}) ;{ }^{13} \mathrm{C}$ NMR $\left(100 \mathrm{MHz}, \mathrm{CDCl}_{3}\right) \delta 197.1,156.7,139.7,128.7,128.1$, 127.8, 125.4, 116.1, 105.0, 100.9, 49.4, 48.0, 44.1, 41.8, 14.4; IR (neat) 2923, 1625, 1429, 1330, 1129, 968, $700 \mathrm{~cm}^{-1}$; HRMS calcd for $[\mathrm{M}] \mathrm{C}_{17} \mathrm{H}_{16} \mathrm{Br}_{2} \mathrm{~N}_{2} \mathrm{O} 437.9579$, found 437.9576.

2-((S)-6,7-Dibromo-2-((R)-1-(naphthalene-1-yl)ethyl)-1oxo-1,2,3,4-tetrahydropyrrolo[1,2-a]pyrazin-4-yl)acetaldehyde (4b). Dichloromethane $(2 \mathrm{~mL}, 0.1 \mathrm{M})$, acrolein $(40 \mu \mathrm{L}$, $0.6 \mathrm{mmol}$ ) and Hoveyda-Grubbs 2 nd generation catalyst $(6.25$ $\mathrm{mg}, 0.01 \mathrm{mmol})$ were added to a $10 \mathrm{~mL}$ round-bottomed flask charged with $(R)-N$-allyl-4,5-dibromo- $N$-(1-(naphthalen-1-yl) ethyl)-1H-pyrrole-2-carboxamide (3b, $98 \mathrm{mg}, 0.2 \mathrm{mmol})$ at room temperature and the mixture was stirred for 2 hours. At this point of time, additional Hoveyda-Grubbs 2 nd generation catalyst $(6.25 \mathrm{mg}, 0.01 \mathrm{mmol})$ was added in the reaction mixture and the mixture was continuously stirred for 2 hours. After that time, the solution was concentrated to dryness and purified by flash chromatography $\left(\mathrm{SiO}_{2}, 20 \%\right.$ EtOAc/hexanes) to provide $\mathbf{4 b - I}$ as major diastereomer in $43 \%$ yield ( $42 \mathrm{mg}$, a yellow solid), and $\mathbf{4 b}$-II as minor diastereomer in 13\% yield (13 mg, a brown solid), respectively (total yield: $56 \%$ ):

4b-I as Major Diastereomer: $\mathrm{mp} 101-103{ }^{\circ} \mathrm{C} ;[\alpha]_{\mathrm{D}}^{20}-20.1(\mathrm{c}$

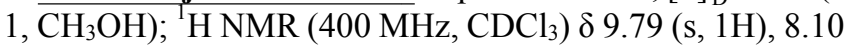
$(\mathrm{d}, J=8.0 \mathrm{~Hz}, 1 \mathrm{H}), 7.86-7.82(\mathrm{~m}, 2 \mathrm{H}), 7.54-7.46(\mathrm{~m}, 4 \mathrm{H}), 7.07$ $(\mathrm{s}, 1 \mathrm{H}), 6.71(\mathrm{q}, J=6.8 \mathrm{~Hz}, 1 \mathrm{H}), 4.61-4.58(\mathrm{~m}, 1 \mathrm{H}), 3.28(\mathrm{dd}$, $J=14.4,1.6 \mathrm{~Hz}, 1 \mathrm{H}), 2.99$ (dd, $J=18.8,10.8 \mathrm{~Hz}, 1 \mathrm{H}), 2.87$ (ddd, $J=14.4,4.0,1.6 \mathrm{~Hz}, 1 \mathrm{H}), 2.75-2.70(\mathrm{~m}, 1 \mathrm{H}), 1.64(\mathrm{~d}, J=$ $7.2 \mathrm{~Hz}, 3 \mathrm{H}) ;{ }^{13} \mathrm{C} \mathrm{NMR}\left(100 \mathrm{MHz}, \mathrm{CDCl}_{3}\right) \delta 198.0,156.2,134.1$, 133.7, 131.6, 129.2, 128.7, 127.3, 126.1, 125.4, 125.0, 124.9, $123.4,116.3,104.9,101.2,48.2,46.4,45.0,43.3,17.2$; IR (neat) 2924, 1725, 1629, 1427, 1330, 1129, $780 \mathrm{~cm}^{-1}$; HRMS calcd for $[\mathrm{M}] \mathrm{C}_{21} \mathrm{H}_{18} \mathrm{Br}_{2} \mathrm{~N}_{2} \mathrm{O}_{2}$ 487.9735, found 487.9733: 
4b-II as Minor Diastereomer: $\mathrm{mp} 66-68^{\circ} \mathrm{C}$; $[\alpha]_{\mathrm{D}}^{20}-8.7$ ( c 1, $\left.\mathrm{CH}_{3} \mathrm{OH}\right) ;{ }^{1} \mathrm{H}$ NMR $\left(400 \mathrm{MHz}, \mathrm{CDCl}_{3}\right) \delta 8.06(\mathrm{~d}, J=8.4 \mathrm{~Hz}$, $1 \mathrm{H}), 7.97(\mathrm{~s}, 1 \mathrm{H}), 7.86-7.81(\mathrm{~m}, 2 \mathrm{H}), 7.57-7.47(\mathrm{~m}, 4 \mathrm{H}), 7.06$ $(\mathrm{s}, 1 \mathrm{H}), 6.67(\mathrm{q}, J=6.8 \mathrm{~Hz}, 1 \mathrm{H}), 4.43-4.40(\mathrm{~m}, 1 \mathrm{H}), 3.67$ (ddd, $J=13.2,4.0,1.6 \mathrm{~Hz}, 1 \mathrm{H}), 3.17(\mathrm{dd}, J=13.2,1.6 \mathrm{~Hz}, 1 \mathrm{H}), 2.00$ (ddd, $J=18.8,2.8,1.6 \mathrm{~Hz}, 1 \mathrm{H}), 1.74-1.67(\mathrm{~m}, 1 \mathrm{H}), 1.69(\mathrm{~d}, J=$ $6.8 \mathrm{~Hz}, 3 \mathrm{H}) ;{ }^{13} \mathrm{C} \mathrm{NMR}\left(100 \mathrm{MHz}, \mathrm{CDCl}_{3}\right) \delta 196.4,156.6,134.7$, 133.7, 131.2, 129.0, 128.9, 127.2, 126.2, 125.3, 125.2, 123.6, 116.2, 105.0, 100.8, 48.3, 46.6, 44.0, 41.8, 15.2; IR (neat) 2924, 1720, 1644, 1427, 1329, 1128, $781 \mathrm{~cm}^{-1}$; HRMS calcd for [M] $\mathrm{C}_{21} \mathrm{H}_{18} \mathrm{Br}_{2} \mathrm{~N}_{2} \mathrm{O}_{2} 487.9735$, found 487.9732.

(S)-6,7-Dibromo-4-(2-hydroxyethyl)-2-((R)-1-(naphthalene-1-yl)ethyl)-3,4-dihydropymolo $[1,2-a]$ pyrazin-1(2H)-one (5). Into a $10 \mathrm{~mL}$ flask was placed the tandem product $\mathbf{4 b}$ (170 $\mathrm{mg}, 0.346 \mathrm{mmol})$ in ethanol $(3.5 \mathrm{~mL}, 0.1 \mathrm{M})$. The solution was cooled to $0{ }^{\circ} \mathrm{C}$, and sodium borohydride $(16 \mathrm{mg}, 0.415 \mathrm{mmol})$ was added in a single portion. After $30 \mathrm{~min}$, the reaction was quenched by the dropwise addition of $1 \% \mathrm{HCl}$ solution. The mixture was poured into ethyl acetate. The combined organic layers were washed with saturated $\mathrm{NaHCO}_{3}$ and brine, and dried over $\mathrm{Na}_{2} \mathrm{SO}_{4}$. Concentration and purification by flash chromatography $\left(\mathrm{SiO}_{2}, 40 \% \mathrm{EtOAc} /\right.$ hexanes $)$ provided 5 in 94\% yield $(160 \mathrm{mg})$ as a white solid: $\mathrm{mp} 176-178{ }^{\circ} \mathrm{C} ;[\alpha]_{\mathrm{D}}^{20}-32.9(c 0.9$, $\left.\mathrm{CH}_{3} \mathrm{OH}\right) ;{ }^{1} \mathrm{H} \mathrm{NMR}\left(400 \mathrm{MHz}, \mathrm{CDCl}_{3}\right) \delta 8.06(\mathrm{~d}, J=8.0 \mathrm{~Hz}$, $1 \mathrm{H}), 7.84-7.81(\mathrm{~m}, 2 \mathrm{H}), 7.57-7.44(\mathrm{~m}, 4 \mathrm{H}), 7.05(\mathrm{~s}, 1 \mathrm{H}), 6.71$ $(\mathrm{q}, J=6.8 \mathrm{~Hz}, 1 \mathrm{H}), 4.32-4.28(\mathrm{~m}, 1 \mathrm{H}), 3.77-3.74(\mathrm{~m}, 2 \mathrm{H}), 3.30$ (dd, $J=14.0,1.2 \mathrm{~Hz}, 1 \mathrm{H}), 2.81$ (dd, $J=14.0,4.4 \mathrm{~Hz}, 1 \mathrm{H}), 2.04-$ $1.94(\mathrm{~m}, 2 \mathrm{H}), 1.85-1.77(\mathrm{~m}, 1 \mathrm{H}), 1.73(\mathrm{~d}, J=6.4 \mathrm{~Hz}, 3 \mathrm{H}) ;{ }^{13} \mathrm{C}$ NMR (100 MHz, $\left.\mathrm{CDCl}_{3}\right) \delta 156.4,134.4,133.6,131.7,129.1$, $128.7,127.2,126.1,125.1,124.9,124.8,123.4,115.7,105.4$, $100.5,59.1,51.3,46.7,43.8,34.8,17.0$; IR (neat) 3425, 2926, $1729,1627,1429,1285,1129,1055,745 \mathrm{~cm}^{-1}$; HRMS calcd for [M] $\mathrm{C}_{21} \mathrm{H}_{20} \mathrm{Br}_{2} \mathrm{~N}_{2} \mathrm{O}_{2} 489.9892$, found 489.9889 .

(S)-6,7-Dibromo-4-(2-hydroxyethyl)-3,4-dihydropyrrolo [1,2-a]pyrazin-1(2H)-one (6). Methanesulfonic acid (159 mg, $1.65 \mathrm{mmol})$ and anisole $(18 \mu \mathrm{L}, 0.165 \mathrm{mmol})$ were added to a stirred solution of $5(81 \mathrm{mg}, 0.165 \mathrm{mmol})$ in nitromethane $(1.7$ $\mathrm{mL}, 0.1 \mathrm{M}$ ), and the mixture was stirred for 4 hours at $120^{\circ} \mathrm{C}$. The reaction mixture was poured into saturated $\mathrm{NaHCO}_{3}$ and extracted twice with dichloromethane. The combined organic layers were dried over $\mathrm{MgSO}_{4}$. Concentration and purification by flash chromatography $\left(\mathrm{SiO}_{2}, 5 \% \mathrm{MeOH} / \mathrm{DCM}\right)$ provided 6 in $80 \%$ yield (45 mg) as a white solid: $\mathrm{mp} 138-139{ }^{\circ} \mathrm{C} ;[\alpha]_{\mathrm{D}}^{20}$ -31.9 (c 1, $\left.\mathrm{CH}_{3} \mathrm{OH}\right) ;{ }^{1} \mathrm{H}$ NMR (400 MHz, $\left.\mathrm{CD}_{3} \mathrm{OD}\right) \delta 6.91(\mathrm{~s}$, $1 \mathrm{H}), 4.60-4.56(\mathrm{~m}, 1 \mathrm{H}), 3.80$ (ddd, $J=13.6,4.0,0.4 \mathrm{~Hz}, 1 \mathrm{H})$, $3.71-3.61(\mathrm{~m}, 3 \mathrm{H}), 1.97-1.95(\mathrm{~m}, 1 \mathrm{H}), 1.87-1.79(\mathrm{~m}, 1 \mathrm{H}) ;{ }^{13} \mathrm{C}$ NMR $\left(100 \mathrm{MHz}, \mathrm{CD}_{3} \mathrm{OD}\right) \delta 161.1,126.1,116.4,108.1,101.2$, 59.3, 53.4, 43.4, 35.5; IR (neat) 3435, 3243, 2922, 1647, 1616, $1544,1424,1334,1053,959,749 \mathrm{~cm}^{-1}$; HRMS calcd for [M] $\mathrm{C}_{9} \mathrm{H}_{10} \mathrm{Br}_{2} \mathrm{~N}_{2} \mathrm{O}_{2}$ 335.9109, found 335.9107.

Acknowledgments. This Research was supported by Kyungpook National University Research Fund, 2008.

\section{References and Notes}

1. For recent reviews of ruthenium-carbene catalyzed olefin metathesis, see: (a) Lozano-Vila, A. M.; Monsaert, S.; Bajek, A.; Ver- poort, F. Chem. Rev. 2010, 110, 4865. (b) Vougioukalakis, G. C.; Grubbs, R. H. Chem. Rev. 2010, 110, 1746. (c) van Otterlo, W. A.; de Koning, C. B. Chem. Rev. 2009, 109, 3743. (d) Samojłowicz, C.; Bieniek, M.; Grela, K. Chem. Rev. 2009, 109, 3708. (e) Burtscher, D.; Grela, K. Angew. Chem. Int. Ed. 2009, 48, 442. (f) Hoveyda, A. H.; Zhugralin, A. R. Nature 2007, 450, 243.

2. For reviews of non-metathetic behavior patterns of metathesisactive ruthenium-carbene catalysts, see: (a) Alcaide, B.; Almendros, P.; Luna, A. Chem. Rev. 2009, 109, 3817. (b) Alcaide, B.; Almendros, P. Chem. Eur. J. 2003, 9, 1259.

3. Seigal, B. A.; Fajardo, C.; Snapper, M. L. J. Am. Chem. Soc. 2005, $127,16329$.

4. (a) Donohoe, T. J.; O'Riordan, T. J. C.; Rosa, C. P. Angew. Chem. Int. Ed. 2009, 48, 1014. (b) Henessian, S.; Giroux, S.; Larsson, A. Org. Lett. 2006, 8, 5481. (c) Finnegan, D.; Seigal, B. A.; Snapper, M. L. Org. Lett. 2006, 8, 2603. (d) Schmidt, B. Chem. Commun. 2004, 742. (e) Sutton, A. E.; Seigal, B. A.; Finnegan, D. F.; Snapper, M. L. J. Am. Chem. Soc. 2002, 124, 13390.

5. (a) Beligny, S.; Eibauer, S.; Maechling, S.; Blechert, S. Angew. Chem. Int. Ed. 2006, 45, 1900. (b) Scholte, A. A.; An, M. H.; Snapper, M. L. Org. Lett. 2006, 8, 4759.

6. Louie, J.; Bielawski, C. W.; Grubbs, R. H. J. Am. Chem. Soc. 2001, 123,11312

7. Fustero, S.; Jiménez, D.; Sánchez-Roselló, M.; del Pozo, C. J. Am. Chem. Soc. 2007, 129, 6700.

8. Fuwa, H.; Noto, K.; Sasaki, M. Org. Lett. 2010, 12, 1636.

9. Chen, J.-R.; Li, C.-F.; An, X.-L.; Zhang, J.-J.; Zhu, X.-Y.; Xiao, W.-J. Angew. Chem. Int. Ed. 2008, 47, 2489.

10. Kim, B. G.; Snapper, M. L. J. Am. Chem. Soc. 2006, 128, 52.

11. (a) Cho, E. J.; Lee, D. Org. Lett. 2008, 10, 257. (b) Lee, D.; Kim, M. Org. Biomol. Chem. 2007, 5, 3418. (c) Kim, M.; Lee, D. J. Am. Chem. Soc. 2005, 127, 18024 .

12. Gavenonis, J.; Arroyo, R. V.; Snapper, M. L. Chem. Commun. 2010, 46, 5692.

13. (a) Seiple, I. B.; Su, S.; Young, I. S.; Lewis, C. A.; Yamaguchi, J.; Baran, P. S. Angew. Chem. Int. Ed. 2010, 49, 1095. (b) When, P. M.; Bois, J. D. Angew. Chem. Int. Ed. 2009, 48, 3802. (c) Imaoka, T.; Iwamato, O.; Noguchi, K.-i.; Nagasawa, K. Angew. Chem. Int. Ed. 2009, 48, 3799. (d) Trost, B. M.; Dong, G. J. Am. Chem. Soc. 2006, 128, 6054. (e) Jacquot, D. E. N.; Lindel, T. Curr. Org. Chem. 2005, 9,1551

14. (a) Hong, S. H.; Wenzel, A. G.; Salguero, T. T.; Day, M. W.; Grubbs, R. H. J. Am. Chem. Soc. 2007, 129, 7961. (b) Hong, S. H.; Sanders, D. P.; Lee, C. W.; Grubbs, R. H. J. Am. Chem. Soc. 2005, 127, 17160. (c) Hong, S. H.; Day, M. W.; Grubbs, R. H. J. Am. Chem. Soc. 2004, 126, 7414.

15. (a) Berlinck, R. G. S.; Kossuga, M. H. Nat. Prod. Rep. 2005, 22 , 516. (b) Faulkner, D. J. Nat. Prod. Rep. 2002, 19, 1. (c) Gribble, G. W. J. Nat. Prod. 1992, 55, 1353.

16. Mancini, I.; Guella, G.; Amade, P.; Roussakis, C.; Pietra, F. Tetrahedron Lett. 1997, 38, 6271

17. Cafieri, F.; Fattorusso, E.; Taglialatela-Scafati, O. J. Nat. Prod. 1998, 61, 122 .

18. (a) Srinivasa Reddy, N.; Venkateswarlu, Y. Biochem. Syst. Ecol. 2000, 28, 1035. (b) Umeyama, A.; Ito, S.; Yuasa, E.; Arihara, S.; Yamada, T. J. Nat. Prod. 1998, 61, 1433.

19. Fattorusso, E.; Taglialatela-Scafati, O. Tetrahedron Lett. 2000 , $41,9917$.

20. Tsuda, M.; Yasuda, T.; Fukushi, E.; Kawabata, J.; Sekiguchi, M.; Fromont, J.; Kobayashi, J. Org. Lett. 2006, 8, 4235.

21. Trost, B. M.; Dong, G. Org. Lett. 2007, 9, 2357.

22. (a) Patel, J.; Pelloux-Léon, N.; Minassian, F.; Vallée, Y. Tetrahedron Lett. 2006, 47, 5561. (b) Patel, J.; Pelloux-Léon, N.; Minassian, F.; Vallée, Y. J. Org. Chem. 2005, 70, 9081.

23. For racemic syntheses of the above bromopyrrolopiperazinone natural products, see: (a) Sun, X.-T.; Chen, A. Tetrahedron Lett. 2007, 48, 3459. (b) Pöverlein, C.; Breckle, G.; Lindel, T. Org. Lett. 2006, 8, 819. (c) Papeo, G.; Antonia, M.; Frau, G.-Z.; Borghi, D.; Varasi, M. Tetrahedron Lett. 2005, 46, 8635. (d) Banwell, M. G.; Bray, A. M.; Willis, A. C.; Wong, D. J. New J. Chem. 1999, 23, 687. 